\title{
Solid-Liquid Interface Shape in Copper Crystals Grown from the Melt by the Czochralski Method
}

\author{
By Tetsuo Inoue*
}

\begin{abstract}
The effects of the growth conditions on the solid-liquid interface shape during the Czochralski growth of $\mathrm{Cu}$ crystals have been investigated. It is found that the interface shape is convex towards the melt in all cases, although the degree of the convexity of the interface varies with the growth conditions; the main results are as follows: (1) The degree of interface convexity increases with a decrease of the crystal radius. (2) The degree of interface convexity increases with an increase of the crucible rotation rate. (3) In a shoulder part of the crystal ingot, where its diameter increases, the degree of interface convexity is high, but it changes to a low value in the next part of the ingot where the diameter is constant. (4) The degree of interface convexity is almost independent of the growth rate. (5) A forced convection by Ar gas near the growing interface has little effect on the solid-liquid interface shape.

Moreover, the effect of the degree of the interface convexity on the crystal perfection has been examined. It is shown that the degree of crystal perfection is probably related to the thermal stress which arises from the convexity of the solid-liquid interface.
\end{abstract}

(Received May 16, 1975)

\section{Introduction}

Shapes of the solid-liquid interface have a large effect on the properties and the degree of perfection of single crystals grown from the melt ${ }^{(1) \sim(7)}$. The existence of a curved interface can produce thermal stresses and a large number of dislocations can be introduced during crystal growth.

In a circular cylinder, if one considers that the solid-liquid interface is convex to the melt and the radial temperature difference $\Delta T=$ $T_{1}-T_{2}$ exists between the center and the surface of the ingot near the interface as shown in Fig. 1, one obtains a simple equation of the radial temperature difference due to the thermal stress ${ }^{(1)}$

$$
\sigma=\alpha E \Delta T
$$

where $\alpha$ is the linear expansion coefficient and $E$ is the Young's modulus. Here $\Delta T$ is given by

$$
\Delta T=G \Delta a
$$

where $G$ is the axial temperature gradient at the center of the ingot near the interface and

* The Research Institute for Iron, Steel and Other Metals, Tohohu University, Sendai 980, Japan.

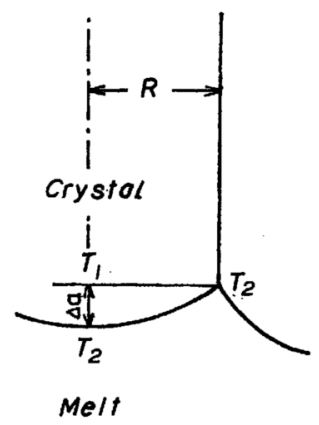

Fig. 1 For illustrating the thermal stress near the convex interface in growing cylindrical crystals.

$\Delta a$ is the deviation from flatness.

From the experimental and theoretical studies $^{(1) \sim(11)}$, it is found that the solidliquid interface shape varies with growth conditions. However, a considerable amount of the work is concerned with materials with low thermal conductivity such as semiconductors $\left(\mathrm{Ge}^{(1) \sim(3)}, \mathrm{Si}^{(4)(5)}\right)$ and oxide (garnet $^{(6)}$, ruby $\left.{ }^{(6)}, \mathrm{TeO}_{2}{ }^{(7)}, \mathrm{Gd}_{3} \mathrm{Ga}_{5} \mathrm{O}_{12}{ }^{(8)}\right)$. The experimental results so far obtained in the Czochralski crystal growth are as follows:

(1) The interface shape varies from convex to concave towards the melt with an increase 
of the pulling rate $\mathrm{r}^{(4)}$.

(2) There is a tendency for the interface to assume a concave shape near the start and a convex one near the end of the ingot, but in the middle part of the ingot where its diameter is nearly constant, the interface remains nearly flat ${ }^{(1)}$.

(3) The interface shape varies from concave towards the melt to flat with a decrease of the crystal radius $^{(3)(7)}$.

(4) The interface shape varies from convex towards the melt to flat with an increase of the crystal rotation rate ${ }^{(5)(6)}$.

The work reported here deals with the solidliquid interface shape in relation to the growth condition and the degree of perfection of $\mathrm{Cu}$ crystals grown by the Czochralski method. The interface shapes have been examined with the quick pulling-up method which has been recently developed by the present author and co-worker ${ }^{(12)}$. The radial temperature difference has been determined with thermocouples grown into pulled $\mathrm{Cu}$ crystals. Some observations have also been made on dislocation structures in pulled $\mathrm{Cu}$ crystals using the etch-pit technique.

\section{Experimental Procedure and Results}

Material used for this study was a re-electrolyzed $\mathrm{Cu}$ metal of $99.999 \%$ purity supplied from Mitsubishi Co. All crystals were grown from the melt in a high-purity graphite crucible by the conventional Czochralski method in an atmosphere of Ar. The furnace was provided with a graphite resistance heater and the temperature was monitored by a total radiation pyrometer which activated the PID controller.

$\mathrm{Cu}$ single crystals were grown under various conditions and the shape of the growing interface was checked by separating the crystal from the melt at an acceleration of $\sim 5000$ $\mathrm{cm} / \mathrm{sec}^{2(12)}$. In all cases, the exposed interfaces were convex to the melt. The deviation from flatness $\Delta a$ (see Fig. 1) was measured with a rule and the degree of convexity, defined by the ratio of $\Delta a$ to crystal radius accross the interface $R$, was determined. All crystals were grown from seed crystals about $5 \mathrm{~mm}$ in diameter in the $\langle 111\rangle$ direction. The results obtained are shown in Fig. 2, which shows that the shape of the solid-liquid interface varies with the growth condition in the following ways:

(1) When the constant diameter crystals were grown at $1 \mathrm{~mm} / \mathrm{min}$ with crucible rotation rate $3 \mathrm{rpm}, \Delta a / R$ became larger for the smaller diameter crystals (Fig. 2(a)).

(2) When the crystals $20 \mathrm{~mm}$ in diameter were grown at $1 \mathrm{~mm} / \mathrm{min}, \Delta a / R$ increased with an increase of the crucible rotation rate from 3 to $9 \mathrm{rpm}$ (Fig. 2(b)).

(3) When the crystal diameter was increased 5 to $20 \mathrm{~mm}$ during its initial $\sim 25 \mathrm{~mm}$ growth at the pulling rate $1 \mathrm{~mm} / \mathrm{min}$ with crucible rotation rate $3 \mathrm{rpm}$ and afterwards held constant, $\Delta a / R$ in the shoulder part of the crystal ingot was large while that in the constant diameter part was small and remained constant (Fig. 2(c)).

(4) When the crystals $20 \mathrm{~mm}$ in diameter were grown at pulling rates varying from 1 to $5 \mathrm{~mm} / \mathrm{min}$ with a crucible rotation rate $3 \mathrm{rpm}$, $\Delta a / R$ remained unchanged (Fig. 2(d)).

The growing interface was also examined

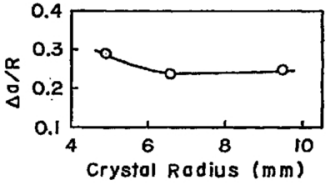

(a)

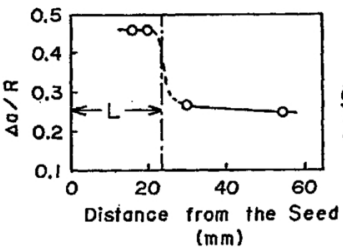

(c)

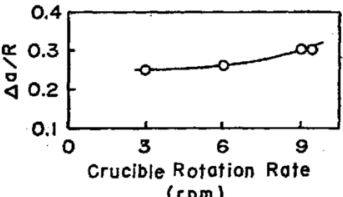

(b)

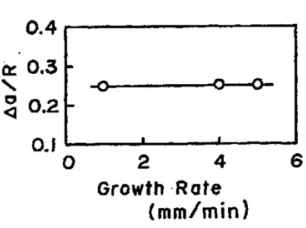

(d)
Fig. 2 Variation of $\Delta a / R$ with growth conditions. All crystals were grown from the seed crystals $\sim 5 \mathrm{~mm}$ in diameter in $\langle 111\rangle$ direction. (a) Crystal radius $R$ was changed from 4.9 to $9.5 \mathrm{~mm}$, growth rate $V=1 \mathrm{~mm} / \mathrm{min}$ and crucible rotation rate $\omega=$ $3 \mathrm{rpm}$. (b) The $\omega$ was changed from 3 to $9 \mathrm{rpm}$, $V=1 \mathrm{~mm} / \mathrm{min}$ and $R=10 \mathrm{~mm}$. (c) The crystal was initially grown with increasing the diameter from 5 to $20 \mathrm{~mm}$ in the region $L$ and held constant afterwards. $V=1 \mathrm{~mm} / \mathrm{min}$ and $\omega=3 \mathrm{rpm}$. (d) The $V$ was changed from 1 to $5 \mathrm{~mm} / \mathrm{min}$. $\omega=3 \mathrm{rpm}$ and $R=10 \mathrm{~mm}$. 


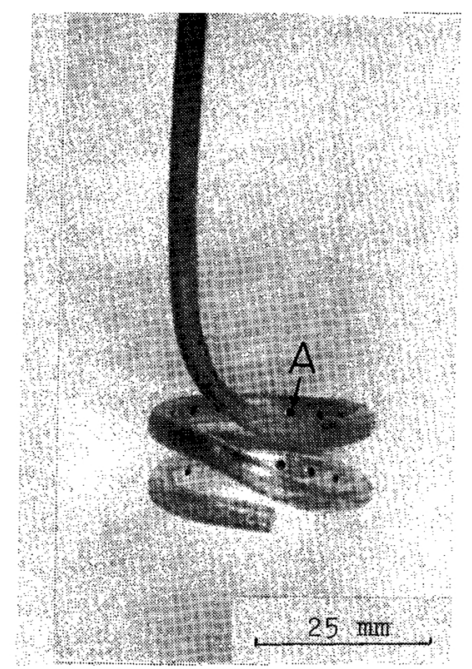

Photo. 1 Argon gas jet nozzle for cooling the crystal surface, which was made of copper tube of $5 \mathrm{~mm}$ diameter. A denotes one of the holes from which the gas blow out.

with the crystals grown in a current of $\mathrm{Ar}$ blown near the growing front. The Ar gas nozzle was of a two-turn spiral type as shown in Photo. 1. Cu crystals $20 \mathrm{~mm}$ in diameter were pulled through this nozzle in a current of $\mathrm{Ar}$ at growth rate $1 \mathrm{~mm} / \mathrm{min}$ with crucible rotation rate $3 \mathrm{rpm}$. The flow rates of Ar were 2 and $8 \mathrm{l} / \mathrm{min}$. It was found that the $\Delta a / R$ values were nearly the same as those obtained in an enclosed atmosphere of Ar. This means that the heat flow in crystal growth is not so much altered by a forced convection of ambient gas near the growing interface. In order to verify this point, measurements of the temperature across a growth face were made. Figure 3 shows an experimental arrangement for the temperature determination used. Two thermocouples $\mathrm{Pt}-13 \% \mathrm{Rh} \cdot \mathrm{Pt} 1.6 \mathrm{~mm}$ in diamenter were sheathed by an Inconel tube and were inserted into holes of the center $\mathrm{A}$ and the periphery B $1 \mathrm{~mm}$ inside the surface in the same horizontal plane of the seed crystal $20 \mathrm{~mm}$ in diameter, respectively. To avoid contamination, thermocouples were used only a few times. The differential output from the thermcouples were continuously recorded during the crystal growth. The crystals were pulled at $0.7 \mathrm{~mm} / \mathrm{min}$ with crucible rotation rate $3 \mathrm{rpm}$ under Ar flow of 2 and $8 \mathrm{\ell} / \mathrm{min}$.

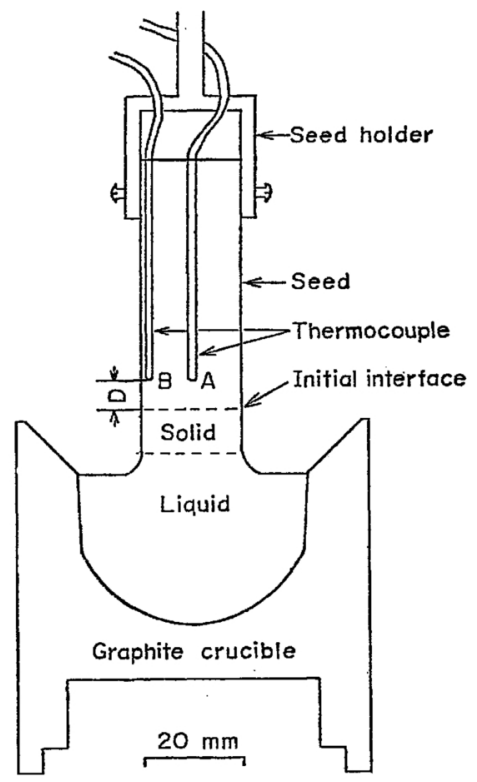

Fig. 3 Arrangement for measuring the radial temperature difference during crystal growth. $D$ denotes the distance between the thermocouples and the initial interface.

Figure 4 shows a plot of the temperature difference between two thermocouples $\Delta T=$ $T_{A}-T_{B}$ against the growth distance. In this figure zero on the abscissa is taken at an initial position of the thermocouples and I represents the assumed growing interface which is apart $\sim 5 \mathrm{~mm}$ from it. It was seen that the curves for both crystals coincided with each other within an experimental error and the radial temperature gradient changed sign near the interface. Heat flow lines and isotherms in the $\mathrm{Cu}$ crystal growth are expected to be as shown in Fig. 5.

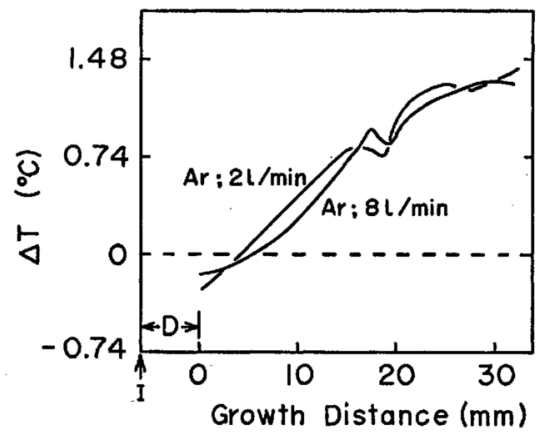

Fig. 4 Variation of $\Delta T$ with growth distance under a forced convection of Ar gas near the interface. 


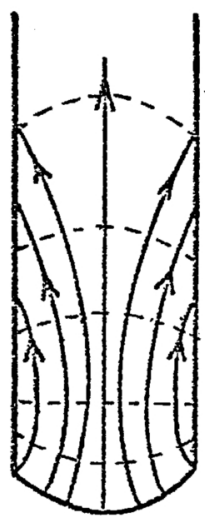

Fig. 5 Heat flow lines and isotherms in $\mathrm{Cu}$ crystal growth for convex interface. Full line: heat flow, dashed line: isotherms.

Finally, two $\mathrm{Cu}$ crystals $20 \mathrm{~mm}$ in diameter were pulled at $1 \mathrm{~mm} / \mathrm{min}$ in an enclosed atmosphere of $\mathrm{Ar}$ in order to examine the variation of crystal perfection with the interface shape. The crucible rotation rates were $3 \mathrm{rpm}$ for Crystal I and $9 \mathrm{rpm}$ for Crystal II which enabled the $\Delta a / R 0.25$ and 0.3 to be found, respectively (see Fig. 2(b)). Two samples from a central portion of each crystals were cut perpendicular to the $\langle 111\rangle$ growth axis with an acid cutter. The (111) surface was treated in the usual way for dislocation etching $^{(13)}$ and the etch pit distributions were observed by means of an optical microscope. The density of randomly distributed dislocation was found to be $\sim 2 \times 10^{5} / \mathrm{cm}^{2}$ for Crystal I with a low $\Delta a / R$ value and to be $\sim 4 \times 10^{5}$ / $\mathrm{cm}^{2}$ for Crystal II with a high $\Delta a / R$ value, while the subboundary length was nearly the same $\sim 1.8 \times 10 \mathrm{~cm} / \mathrm{cm}^{2}$ for both crystals. It is to be added that data were obtained by an etch pit count on about 20 areas on each specimens and the final means were presented.

\section{Discussion}

It has been shown that the shape of the growing interface of copper crystals grown from the melt is convex to the melt and that it depends on the crystal radius and the crucible rotation rate but not on the growth rate and the forced convection of $\mathrm{Ar}$ near the interface. Furthermore, the observation of dislocation structure of grown crystals indicates that the crystal perfection can be related with the degree of interface convexity.

Arizumi and Kobayashi ${ }^{(9)}$ showed theoretically that the rise of the crucible temperature changed the interface from convex to concave towards the melt and the growth rate dependence of the interface shape was inverse to the crucible temperature dependence. Milvidskii ${ }^{(4)}$ also showed experimentally that the interface shape varied from convex to concave towards the melt as the growth rate increased. However, the interface shape was almost independent of the growth rate for $\mathrm{Cu}$ in this experiment (see Fig. 2(d)). On the other hand, the author has found for $\mathrm{KCl}$ crystals $\uparrow$ that the interface shape changes from convex to concave towards the melt with an increase in growth rate. From these results, it can be considered that the growth rate has an important effect on the interface shape for semiconductor crystals or ionic crystals with low thermal conductivity, but it is unimportant for metal crystals such as $\mathrm{Cu}$ with high thermal conductivity.

The dependency of the interface shape on the crystal radius for $\mathrm{Cu}$ crystals (see Fig. 2(a)) agrees well with the experimental results for Ge crystals by Rosi ${ }^{(3)}$ and for $\mathrm{Gd}_{3} \mathrm{Ga}_{5} \mathrm{O}_{12}$ crystals by Sakai et al. ${ }^{(8)}$

The experimental studies of the effect of the crucible rotation on the interface shape have not been reported. However, Kobayashi and Arizumi $^{(11)}$ studied this problem for $\mathrm{Ge}$ crystals theoretically and postulated the following. As the crucible rotation rate increases, the downwards melt flow becomes strong, by which the temperature profile is altered in such a manner that the isotherms may be slightly displaced downward. Then, the degree of convexity of the interface may increase with an increase of the crucible rotation rate. The present results can be interpreted qualitatively by this concept.

Regarding the effect of the crystal length on the interface shape, Billig( ${ }^{(1)}$ reported for $\mathrm{Si}$ crystals that the shape varied from concave to convex towards the melt with an increase of the crystal length, but such a tendency was

$\dagger$ Details will be reported in the near future. 
not observed in this experiment (see Fig. 2(c)). The reason is not clear at present.

Finally, a rough estimate of the thermal stress will be made for Crystal I and Crystal II which were grown for examining the effect of the degree of the interface convexity on the crystal perfection using the method shown in Fig. 1. The radial temperature difference $\Delta T$ which can be estimated from the curvature of the interface, is $1.7^{\circ} \mathrm{C}$ for the Crystal I and $2.1^{\circ} \mathrm{C}$ for the Crystal II. Substituting these vaules of $\Delta T$, the linear expansion coefficient $\alpha=16.8 \times 10^{-6} \mathrm{deg}^{-1}$ and Young's modulus $E=11.4 \times 10^{3} \mathrm{~kg} / \mathrm{mm}^{2}$ into eq. (1) yields the thermal stress $\sigma \sim 330 \mathrm{~g} / \mathrm{mm}^{2}$ for the crystal I and $\sim 400 \mathrm{~g} / \mathrm{mm}^{2}$ for the Crystal II, respectively. As mentioned in II, the density of random dislocations was $\sim 2 \times 10^{5} / \mathrm{cm}^{2}$ for Crystal I and $\sim 4 \times 10^{5} / \mathrm{cm}^{2}$ for Crystal II, while the subboundary length was nearly the same for both crystals. This low density of dislocations in Crystal I is probably related to the relatively low value of thermal stress originating from the convexity of the solidliquid interface.

\section{Summary}

The effects of the growth conditions on the solid-liquid interface shape during the Czochralski growth of $\mathrm{Cu}$ crystals have been investigated and the relation between the interface shape and the crystal perfection has also been examined. The results obtained are summarized as follows.

(1) The interface shape is convex towards the melt in each growth conditions. However, the degree of the interface convexity changes with the growth condition as follows: (a) The degree of the interface convexity increases with a decrease of the crystal radius (see Fig. 2(a)). (b) The degree of interface convexity increases with an increase of the crucible rotation rate (see Fig. 2(b)). (c) In the shoulder part of the ingot where its diameter is increasing, the degree of interface convexity is high, but it changes to a low value and remains constant in the next part where the diameter is constant (Fig. 2(c)). (d) The degree of interface convexity is almost independent of the growth rate (Fig. 2(d)). (e) A forced convection by $\mathrm{Ar}$ gas near the growing front has little effect on the radial temperature difference in the solid as well as the solid-liquid interface shape.

(2) The dislocation density increases as the degree of the interface convexity increases.

\section{Acknowledgment}

The author would like to thank Professor J. Watanabé for suggesting this problem and for stimulating interest in it.

\section{REFERENCES}

(1) E. Billig: Proc. Roy. Soc., A235 (1956), 37.

(2) I. G. Cressel and J. A. Powell: Progress in Semiconductors, Vol. 2, Heywood, London, (1957), p. 139.

(3) F. D. Rosi: RCA Rev., 19 (1959), 349.

(4) M. G. Milvidskii and B. I. Golovin: Soviet Physics-Solid State, 4 (1961), 737.

(5) J. H. Braun and R. A. Pellin: J. Electrochem. Soc., 108 (1961), 969.

(6) B. Cockayne, M. Chesswas and D. B. Gasson: J. Materials Science, 4 (1969), 450.

(7) N. Miyazawa and Y. Iwasaki: Japan J. Appl. Phys., 9 (1970), 441.

(8) M. Sakai, T. Fukuda and H. Tominaga: Journal of the Japanese Association of Crystal Growth, 1 (1974), 11.

(9) T. Arizumi and N. Kobayashi: Japan J. Appl. Phys., 8 (1967), 1091.

(10) N. Kobayashi and T. Arizumi: Japan J. Appl. Phys., 9 (1970), 361.

(11) N. Kobayashi and T. Arizumi: Japan J. Appl. Phys., 9 (1970), 1255.

(12) T. Inoue, J. Watanabé and M. Yamamoto: Trans. JIM, 13 (1972), 370.

(13) K. Marukawa: Japan J. Appl. Phys., 4 (1967), 944. 